Europhys. Lett., 12 (1), pp. 51-55 (1990)

\title{
Finite-Size Effects in the Transition from Hexagons to Rolls in Convective Systems.
}

\author{
C. PÉrez-García $\left(^{*}\right)\left({ }^{\S}\right)$, E. Pampaloni $(* *)$ and S. Ciliberto $(* *)$ \\ (*) Departamento de Física, Facultad de Ciencias, Universidad de Navarra \\ 31080 Pamplona, Navarra, Spain \\ (**) Istituto Nazionale di Ottica - Largo E. Fermi 6, 50125 Arcetri, Firenze, Italia
}

(received 17 January 1990; accepted 15 February 1990)

PACS. 47.20 - Hydrodynamic stability and instability.

PACS. 47.25 - Turbulent flows, convection, and heat transfer.

\begin{abstract}
The transition thresholds between hexagons and rolls in convective patterns are obtained in the framework of the amplitude equations. We show that the discrepancies between the theoretical thresholds, calculated for unbounded systems, and the experimental ones, made in finite containers, can be partially corrected by a phenomenological argument. The finite-size effects are responsible for the decreasing in the efficiency of the heat transport across the cell. Using this fact we are able to approach the calculated thresholds to those observed in real experiments.
\end{abstract}

The transition between different symmetries in convective patterns is the subject of some current studies [1]. The transition between hexagons and rolls appears: $a$ ) in fluids with temperature-dependent transport coefficients (non-Boussinesq conditions) [2-8], b) when the mean temperature in the cell varies (increases or decreases) linearly in time [9], c) in fluids with a temperature-dependent surface tension (Bénard-Marangoni convection) $[10,11]$ and $d)$ by temperature modulations in the convective cell [12-14].

These studies show that the transition is, in general, smooth and hysteretic, but some discrepancies between theoretical predictions and experimental measurements are always present [6-8]. Theoretical studies are made for horizontal unbounded layers of liquid [3], while experiments are in convective cells with a finite aspect ratio $\Gamma$ ( $I$ is the ratio between a horizontal characteristic length and the layer depth $d$ )). Some experimental works are based on laser-Doppler anemometry (local) [5] or on calorimetric data (global) [6, 7]. Using a laser deflection technique [15], which gives local information on the temperature field, it has been shown recently that it is possible to recover global features of the pattern from these local measurements $[8,16]$.

The purpose of the present letter is to show that finite-size effects can be taken into account by simple phenomenological corrections of the theoretical transition thresholds in

(\$) Also at Departamento de Física, Universitat Autònoma de Barcelona, 08193 Bellaterra, Catalonia, Spain. 
terms of amplitude equations and experimental data. In the case of an hexagonal pattern these equations read as [17]

$\tau_{0} \frac{\partial A_{i}}{\partial t}=\left\{\varepsilon+\xi_{0}^{2}\left[\frac{\partial}{\partial x_{i}}-\frac{i}{2 k_{\mathrm{c}}} \frac{\partial^{2}}{\partial y_{1}^{2}}\right]^{2}\right\} A_{i}+a A_{i+1}^{*} A_{i+2}^{*}-b\left[\sum_{i \neq j}\left|A_{j}\right|^{2}\right] A_{i}-c\left|A_{i}\right|^{2} A_{i}(i=i \bmod 3)$,

where $\varepsilon=\left(\Delta T-\Delta T_{\mathrm{c}}\right) / \Delta T_{\mathrm{c}}$ is the normalized Rayleigh number and $\Delta T_{\mathrm{c}}$ denotes the critical temperature difference across the convective layer. This last parameter, as well as the relaxation time $\tau_{0}$, the correlation length and the critical wave number $k_{\mathrm{c}}$ can be calculated with a linear analysis. Instead, the coefficient $a, b$ and $c$ come from a nonlinear analysis. However, we will show in the following that they are accessible simply by heat flow measurements.

The two stationary and homogeneous solutions of (1) are

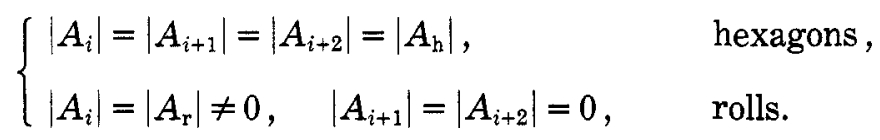

These correspond to the two parabolas in fig. 1 obtained from (1) for these two cases. A linear stability analysis of perturbations around these stationary and homogeneous solutions show that the hexagons are stable in the interval $\varepsilon_{\mathrm{s}} \leqslant \varepsilon \leqslant \varepsilon_{\mathrm{h}}$ while the rolls are stable when $\varepsilon \geqslant \varepsilon_{\mathrm{r}}$, where $\varepsilon_{\mathrm{s}}, \varepsilon_{\mathrm{h}}$ and $\varepsilon_{\mathrm{r}}$ are the following transition thresholds (see also fig. 1):

$$
\begin{cases}\varepsilon_{\mathrm{s}}=-\frac{a^{2} c}{4(2 b+c)} & \text { hexagons } \rightarrow \text { no convection, } \\ \varepsilon_{\mathrm{h}}=\frac{a^{2}(b+2 c)}{(b-c)^{2}} & \text { hexagons } \rightarrow \text { rolls }, \\ \varepsilon_{\mathrm{r}}=\frac{a^{2} c}{(b-c)^{2}} & \text { rolls } \rightarrow \text { hexagons } .\end{cases}
$$

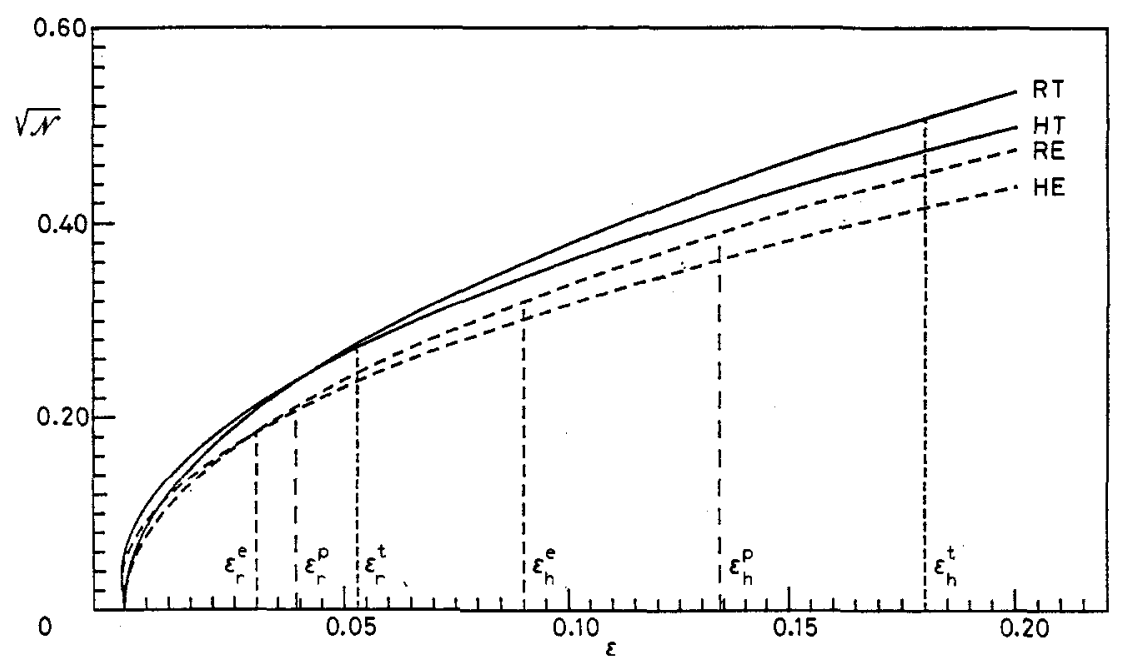

Fig. 1. - Stationary and homogeneous solutions for the amplitude equations (1) for an unbounded system (full line) of rolls (RT) and hexagons (HT). Dashed lines correspond, instead, to a bounded system of rolls (RE) and hexagons (HE) (see text). The transition thresholds $\varepsilon_{\mathrm{r}}$ (rolls $\Rightarrow$ hexagons) and $\varepsilon_{\mathrm{h}}$ (hexagons $\Rightarrow$ rolls) are given. $(\mathrm{t}=$ theoretical; $\mathrm{e}=$ experimental and $\mathrm{p}=$ phenomenological). 
As a consequence, two hysteresis cycles can be distinguished. One between the conducting (rest) state and hexagonal motions, in the interval $\varepsilon_{\mathrm{s}} \leqslant \varepsilon \leqslant 0$, the second one between hexagons and rolls in the interval $\varepsilon_{\mathrm{r}} \leqslant \varepsilon \leqslant \varepsilon_{\mathrm{h}}$. The existence of two transitions (one from rest state to hexagons and the other from hexagons to rolls) and two hysteretic loops have been observed in convection under non-Boussinesq conditions [5, 7, 8]. Some of these features are also observed in the case of a mean temperature linearly increasing in time [9] and in the case of temperature modulation [14]. However, in all these cases some discrepancies between theoretical and experimental results exist. Usually it is argued that these discrepancies are due to the finite-size effects [6].

With a suitable normalization the nondimensional heat flow $\mathscr{N}$ can be written in the form [18]

$$
\mathscr{N} \equiv \frac{(N-1) R}{R_{\mathrm{c}}}=\frac{1}{S} \int \mathrm{d} x \mathrm{~d} y \sum_{i=1}^{3}\left|A_{i}\right|^{2}
$$

where $N$ is the Nusselt number, the ratio between the total heat flow and the conductive heat flow, and $S$ the horizontal area of the convective pattern. For hexagons the nondimensional heat flow $\mathscr{N}$ gives

$$
\mathscr{N}_{\mathrm{h}}=3\left|A_{\mathrm{h}}\right|^{2}=\frac{3}{C}\left\{\frac{a^{2}}{2 C}+\varepsilon+\frac{a}{2 C} \sqrt{a^{2}+4 C \varepsilon}\right\},
$$

where $C=2 b+c$, while one has for rolls

$$
\mathscr{N}_{\mathrm{r}}=\left|A_{\mathrm{r}}\right|^{2}=\varepsilon / c
$$

By a suitable fitting of experimental data with expressions (5), (6) one would recover the three coefficients in the amplitude equations (1): $c$ from the slope of $\mathcal{N}_{\mathrm{r}}$ as a function of $\varepsilon$ for rolls and $b$ and $a$ from the fitting of the heat flow for hexagons with eq. (5). Although this procedure is theoretically possible, some difficulties appear in practice, especially in the determination of $a$. The parameter $a$ is linked to the subcritical threshold loop which is difficult to observe $[7,8]$.

From eqs. (3)-(6) it is obvious that the transition thresholds and the slopes in the heat flow curves are linked, because they depend on the three parameters $a, b$ and $c$. As argued by some authors [7], among these parameters the less sensitive to the lateral effects is $a$, because it mainly depends on non-Boussinesq effects. Thus, when the experimental fitting is not sufficiently accurate one can take the theoretical value for $a$.

The important point, now, is that the discrepancies between the theoretical transition thresholds and those obtained in experiments are linked with the discrepancies between the measured heat flows and those calculated theoretically.

The lateral effects act as a multiplicative factor that decreases the efficiency of the heat transport, both for hexagons and for rolls. So, the slopes of the lines $\mathcal{N}_{\mathrm{h}, \mathrm{r}}(\varepsilon)$ must be smaller than that for an unbounded system, as observed in experiments [6-8]. However, the ratio between the slopes of the linear fit must be less affected by lateral walls [7].

For the sake of comparison we take the values of the physical parameters that correspond to a recent experiment [8]. In this experiment the convective fluid was pure water at a mean temperature of $28.3^{\circ} \mathrm{C}(\mathrm{Pr}=5.63$ ) in a cylindrical cell (aspect ratio $\Gamma=20)$. The liquid depth is $d=1.8 \mathrm{~mm}$ and the critical temperature difference across the cell is $\Delta T_{\mathrm{c}}=12.6 \mathrm{~K}$. In these conditions the non-Boussinesq effects are high enough to induce a hexagonal symmetry in the convective pattern and sufficiently small to allow for a perturbative analysis. The 
TABLE I. - Theoretical and experimental values of the linear part $\gamma$ of the nondimensional heat flow $\mathcal{N}$ as a function of $\varepsilon$.

\begin{tabular}{llll}
\hline Method & $\gamma_{\mathrm{h}}$ & $\gamma_{\mathrm{r}}$ & $\gamma_{\mathrm{r}} / \gamma_{\mathrm{h}}$ \\
\hline Theoretical [3] & 1.11 & 1.43 & 1.29 \\
Experimental [8] & $0.86 \pm 0.03$ & $1.13 \pm 0.02$ & $1.31 \pm 0.06$ \\
\hline
\end{tabular}

TABLE II. - Values of the parameters in the amplitude equations (1) obtained with different methods (see the text).

\begin{tabular}{llll}
\hline Method & $a$ & $b$ & $c$ \\
\hline Theoretical [3] & $8.63 \cdot 10^{-2}$ & 1.01 & 0.70 \\
Experimental [8] & - & $1.30 \pm 0.06$ & $0.89 \pm 0.02$ \\
\hline
\end{tabular}

TABLE III. - Transition thresholds with different methods. The values called phenomenological have been obtained after introducing the experimental values of table II in formulae (3) and in the analytical expression of $\varepsilon_{\mathrm{h}} / \varepsilon_{\mathrm{r}}$ (see text for details).

\begin{tabular}{llll}
\hline Method & $\varepsilon_{\mathrm{r}}$ & $\varepsilon_{\mathrm{h}}$ & $\varepsilon_{\mathrm{h}} / \varepsilon_{\mathrm{r}}$ \\
\hline Theoretical [3] & $5.46 \cdot 10^{-2}$ & 0.188 & 3.44 \\
Experimental [8] & $(3.0 \pm 0.1) \cdot 10^{-2}$ & $0.09 \pm 0.005$ & $3.0 \pm 0.3$ \\
Phenomenological & $(3.9 \pm 1.9) \cdot 10^{-2}$ & $0.134 \pm 0.06$ & $3.45 \pm 0.09$ \\
\hline
\end{tabular}

tabulated values of the transport coefficients of water [19] under these conditions can be used to calculate the slopes and the transition thresholds from the Busse's formulae [3].

The theoretical [3] and experimental [8] values for the coefficients $\gamma_{\mathrm{r}}=1 / c, \gamma_{\mathrm{h}}=3 / C$ of the linear part of the heat flow curves $\mathscr{N}_{\mathrm{h}, \mathrm{r}}(\varepsilon)$ are quoted in table I. One notices that the experimental slopes are smaller than the theoretical ones, but the ratio is almost the same in agreement with the phenomenological argument given above. After some algebra it is also possible to calculate the parameters $a, b$ and $c$ of eq. (1) from the formulae in ref. [3]. Experimental slopes allow for the determination of $b$ and $c$, but not of $a$. The corresponding values have been gathered in table II.

The theoretical thresholds for the hexagons $\Leftrightarrow$ rolls transitions are easily calculated from the expressions in ref. [3]. Experimentally these thresholds were determined with the laser deflection technique $[8,16]$. The corresponding values are given in table III. The disagreement between these values is obvious and not surprising, as a consequence of the finite-size effects. However, one can correct this situation simply by introducing the experimental values for the coefficients $b$ and $c$, in eqs. (3) for the thresholds and in the ratio $\varepsilon_{\mathrm{h}} / \varepsilon_{\mathrm{r}}=(b+2 c) / c$ (the theoretical value of $a$ has been used). The result is also given in table III in the row called phenomenological and sketched in fig. 1 . The main conclusion is that, although these phenomenological values and the experimental ones do not coincide exactly, this phenomenological procedure allows to correct the discrepancies due to the finite-size effects more than a $50 \%$.

The corrections proposed here have a global character. Discrepancy still exists between phenomenological and experimental threshold values, but this can be due to the unavoidable presence of defects in real experiments. These localized defects may act as critical nuclei of 
C. PÉREZ-GARCíA et al:: FINITE-SIZE EFFECTS IN THE TRANSITION FROM HEXAGONS ETC.

the unstable phase [20], modifying sensitively the transition thresholds. Finally we hope that the present arguments will be useful for further comparisons in other systems where the hexagons-rolls transition is observed.

We thank P. Coullet and J. LEGA (Nice) for fruitful comments and discussions. This work has been partially supported by the ECC under the contract SCI-0035-C(CD), by the DGICYT (Spanish Government) project CE-89-0002 and by the Gruppo Nazionale di Struttura della Materia (Italy). We also have benefited from an Integrated Action (no. 46) of the Italy-Spain Scientific Cooperation Program.

\section{REFERENCES}

[1] Coullet P. and Huerre P. (Editors), New Trends in Nonlinear Dynamics and Pattern Forming Phenomena: The Geometry of Nonequilibrium (Plenum Press, New York, N.Y.) to be published.

[2] Palm E., J. Fluid Mech., 8 (1960) 183.

[3] Busse F. H., J. Fluid Mech., 30 (1967) 625.

[4] Hoard C. Q., Robertson C. R. and ACrivos A., Int. J. Heat Mass Transfer, 13 (1970) 849.

[5] Dubois M., Berge P. and Wesfreid J. E., J. Phys. (Paris), 39 (1978) 1253.

[6] Ahlers G., J. Fluid Mech., 110 (1980) 297.

[7] WALden R. W. and Ahlers G., J. Fluid Mech., 109 (1981) 89.

[8] Ciliberto S., Pampaloni E. and Pérez-García C., Phys. Rev. Lett., 61 (1988) 1198.

[9] Krishnamurti R., J. Fluid Mech., 33 (1968) 445.

[10] Cerisier P., Jamond C., Pérez-García C. and Pantaloni J., Phys. Fluids, to be published.

[11] Bestehorn M. and PÉrez-García C., Europhys. Lett., 4 (1987) 1365.

[12] Roppo M. N., Davis S. H. and RosenblatT S., Phys. Fluids, 27 (1984) 796.

[13] Hohenberg P. C. and Swift J., Phys. Rev. A, 35 (1987) 3855.

[14] Meyer C. W., Ahlers G. and Cannell D., Phys. Rev. Lett., 59 (1987) 1577.

[15] Ciliberto S., Francini F. and Simonelli F., Opt. Commun., 54 (1985) 381.

[16] Pampaloni P., Ciliberto S. and Pérez-García C., to be published.

[17] Pérez-García C., Ciliberto S. and Pampaloni E., in Quantitative Measures of Complex Dynamical Systems, edited by N. B. AbrahaM and A. Albano (Plenum Press, New York, N.Y.) to be published.

[18] Ahlers G., Cross M., Hohenberg P. C. and Safran S., J. Fluid Mech., 110 (1981) 297.

[19] WEAST R. C. (Editor), Handbook of Chemistry and Physics, 66th ed. (CRC Press, Boca Ratón, Florida) 1985.

[20] Coullet P., Gil L. and Repaux D., Phys. Rev. Lett., 62 (1989) 2957. 